\title{
Low-grade nasopharyngeal papillary adenocarcinoma: a case report and review of the literature
}

This article was published in the following Dove Press journal:

OncoTargets and Therapy

18 May 2016

Number of times this article has been viewed

\author{
Xiaoli Wang ${ }^{1,2}$ \\ Hongjiang Yan' \\ Yijun Luo ${ }^{1,2}$ \\ Tingyong Fan' \\ 'Department of Radiation Oncology, \\ Shandong Cancer Hospital and \\ Institute, Jinan, ${ }^{2}$ School of Medicine \\ and Life Sciences, Shandong Academy \\ of Medical Sciences, University of \\ Jinan, Jinan, People's Republic of China
}

\begin{abstract}
Low-grade nasopharyngeal papillary adenocarcinoma is an extremely rare tumor, with only a limited number of cases reported in the literature. Some published studies have paid more attention to the clinicopathological features of nasopharyngeal adenocarcinoma, while little effort has been made to study the optimal therapeutic strategies. We report about a woman diagnosed with low-grade nasopharyngeal papillary adenocarcinoma. She received the treatment approach that combined transnasal endoscopic surgery to remove the lesion with postoperative radiotherapy for nasal cavity. There was no evidence of recurrence after 4 months of surgery, and further follow-up is being continued. Through this example, we wanted to explore the optimal therapeutic strategies for primary nasopharyngeal adenocarcinomas.
\end{abstract}

Keywords: nasopharyngeal adenocarcinoma, immunohistochemistry, treatment policy, survival and prognosis

\section{Introduction}

Nasopharyngeal carcinoma (NPC) is commonly diagnosed in Southeastern Asia, particularly in southern China. ${ }^{1}$ It originates from the epithelial lining of the nasopharynx and has a variety of pathological subtypes. The vast majority of NPC cases are keratinizing or nonkeratinizing squamous cell carcinoma. ${ }^{2}$ Additional pathological types of NPC, which include adenocarcinoma, lymphoma, sarcoma, and minor salivary gland tumor, constitute $<5 \%$ of all NPC cases. ${ }^{3,6}$ Therefore, primary nasopharyngeal adenocarcinomas (NPACs) are extremely rare, with only a limited number of cases reported in the literature ${ }^{1-3}$ Having obtained written informed consent from the patient and approval of the research ethics board of Shangdong Cancer hospital, we report a case of primary lowgrade nasopharyngeal papillary adenocarcinoma (LGNPPA) in a 36-year-old woman who was treated in our hospital. We also include a review of the literature in order to highlight what we know to date about this rare entity.

\section{Case report}

A 36-year-old Chinese woman presented with a 2-year history of bilateral nasal obstruction and mild postnasal drip. It did not vary in severity during this period. No bloody sputum, epistaxis, tinnitus, otitis media, facial pain, aural fullness, or any other complaint was mentioned. A computed tomography (CT) scan of the head and neck showed bilateral mucosal thickening in the maxillary sinus, a soft tissue density in the ethmoid and sphenoid sinuses, and a soft tissue mass in the nasopharynx (Figure 1). There was no additional relevant medical history. The cervical lymph nodes were not
Correspondence: Tingyong Fan

Department of Radiation Oncology, Shandong Cancer Hospital and Institute, Jiyan Road 440, Jinan 250117, Shandong Province, People's Republic of China Tel/fax +86 53I 6762692 I

Email fanty68II05@163.com 


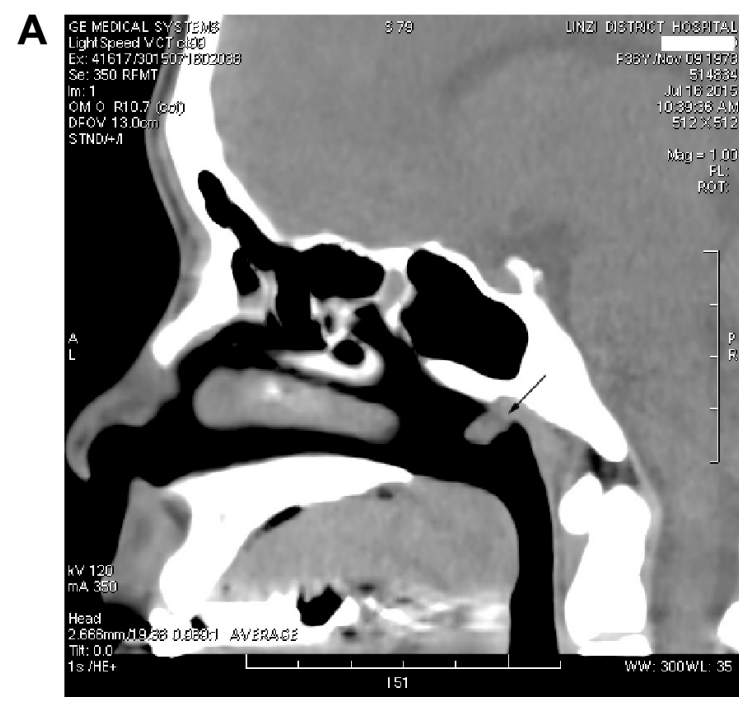

Figure I CT scan: the tumor was identified on the roof of the nasopharynx (arrow) Notes: (A) Sagittal view and (B) coronal view.

Abbreviation: CT, computed tomography.

palpable, and the physical examination findings were also not remarkable, except for nasal septum deviation irregularly. Other investigations were negative, including CT scan and sonography of thyroid.

According to the localized nature of the lesion and the benign appearance, transnasal endoscopic resection was undertaken. In the surgical treatment, a papillary pedicle mass of size $0.7 \mathrm{~cm}$, which was pedunculated and freely mobile, was found on the roof of the nasopharynx. Subsequently, the mass was resected with its stalk. The tumor was completely excised on endoscopy with an adequate surgical margin. Hematoxylin and eosin staining was performed for microscopic examination, and the lesion was characterized by papillary and glandular epithelial proliferation (Figure 2). Postoperative histopathology showed a lowgrade papillary adenocarcinoma, and surgical margin was

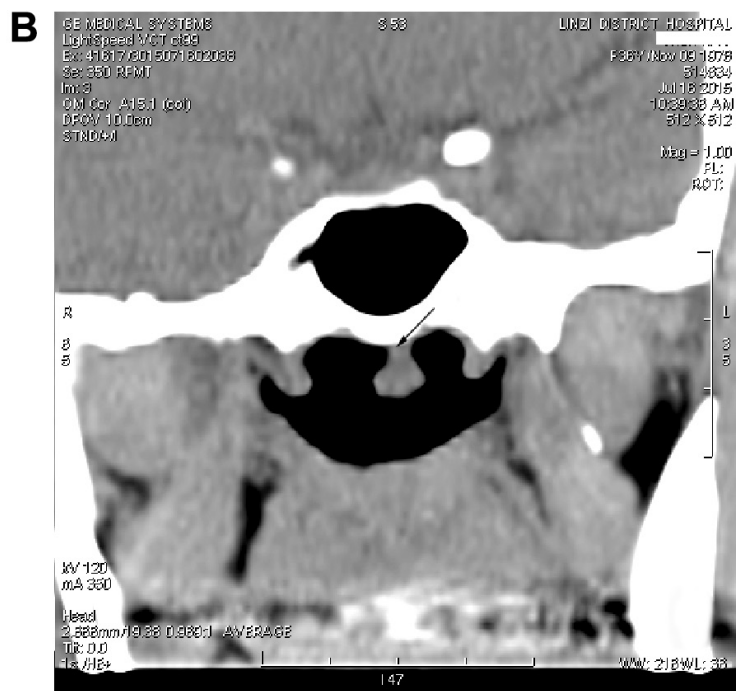

negative. According to the patient's strong will after surgery and the cases reported in the literature, she had accepted postoperative radiotherapy (PORT) for nasal cavity with a total dose of 60 gray in 30 fractions over 6 weeks in our hospital. There was no evidence of recurrence after 4 months of surgery, and further follow-up is being carried out.

\section{Discussion}

The most common malignancy in the nasopharynx is NPC. By and large, the most common nasopharyngeal neoplasm is nonkeratinizing and keratinizing squamous cell carcinomas without glandular differentiation. ${ }^{2}$ Primary NPACs as a group are extremely rare tumors, which are reported to occupy $0.48 \%$ of all types of NPC. ${ }^{3-5}$

Primary NPACs can be classified into two main categories: the conventional or mucosal surface origin type
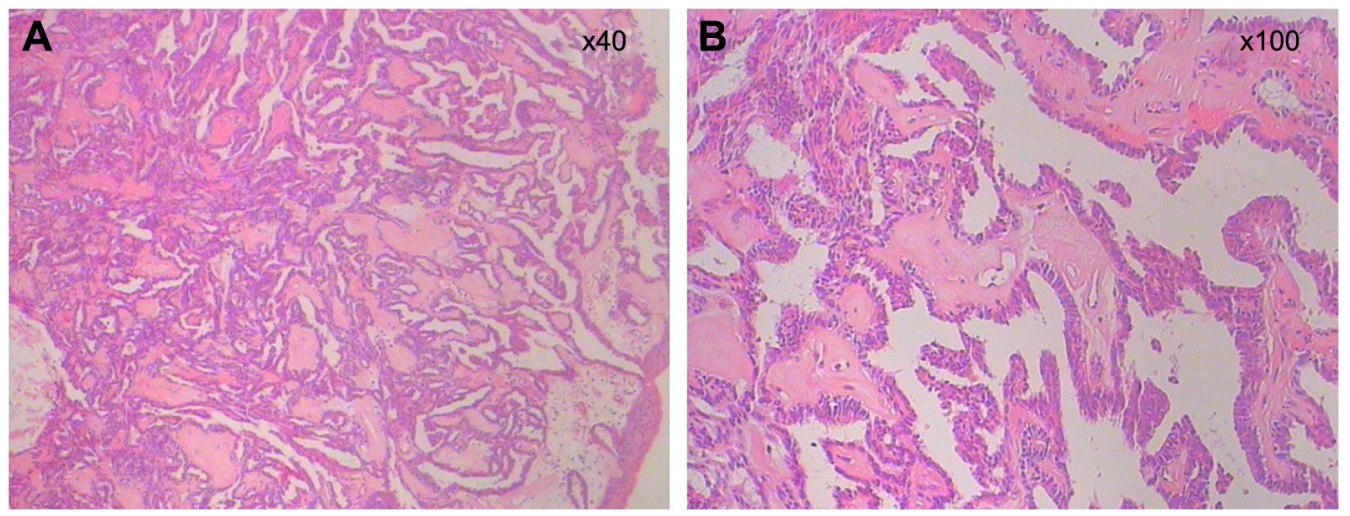

Figure 2 The pictures show typical papillary growth pattern of glandular epithelial cells, which is usually seen in papillary thyroid carcinoma.

Notes: (A) H\&E $\times 40$ and (B) $\mathrm{H} \& \mathrm{E} \times 100$.

Abbreviation: H\&E, hematoxylin and eosin. 
and the salivary gland type, with different morphological features and clinical behavior., ${ }^{2,3-10}$ Nasopharyngeal papillary adenocarcinoma, which belongs to the former category, has been reported in the literature. ${ }^{2}$ A retrospective study first described LGNPPAs as a distinct entity in a series of nine patients in $1988 .^{7}$ There have been several case reports or small series in the literature about this entity after this seminal paper. ${ }^{7,9-12}$ Primary salivary gland-type nasopharyngeal carcinoma belongs to the latter category, which involves two main pathological subtypes: adenoid cystic carcinoma and mucoepidermoid carcinoma. ${ }^{2,9,13}$

The case we presented was LGNPPA, which according to the World Health Organization is defined LGNPPA as phyllodes papillary and glandular structures with exophytic growth characteristics of low-grade adenocarcinoma. ${ }^{8}$ Papillary NPAC is a special type of low-grade malignant carcinoma, and its morphology is similar to papillary thyroid carcinoma, which requires identification with the metastasis of papillary thyroid carcinoma. Thyroid transcription factor-1 (TTF-1) is generally deemed to be specific to the thyroid gland and the lung. However, some reports have occasionally shown positive immunohistochemical reactivity for TTF-1 in nasopharyngeal papillary adenocarcinoma. ${ }^{8,10}$ Therefore, TTF-1 protein might not be a suitable marker for distinguishing between primary or metastatic nasopharyngeal papillary adenocarcinoma. But the negative reactivity for thyroglobulin and CK20 promotes distinguishing metastatic thyroid papillary carcinoma from LGNPPA. ${ }^{10}$ Our case was diagnosed as LGNPPA because both derived from the epithelial surface and had a negative thyroid scan.

Recent and past studies are consistent in revealing the presence of Epstein-Barr virus (EBV) to be closely linked with the development and carcinogenesis of type II and III tumors. ${ }^{2,4}$ EBV DNA as a molecular marker is universally associated with the nonkeratinizing subtype of NPC. ${ }^{14}$ Some studies have detected low-expression levels of EBV markers in the NPAC tissue. ${ }^{2,15}$ Therefore, the relationship between NPACs and EBV is still uncertain. Further examination is still needed because of the extreme rarity of this tumor.

The treatment policy of primary NPACs is mainly chosen according to the clinical stage and histological grade of the tumor. ${ }^{6,16}$ Owing to the rarity of these cancers, the management of the lesions is usually empirically based, and no standard treatment exists for the tumors. Controversy exists as to whether radiotherapy alone, surgical treatment alone, or surgery combined with radiotherapy is the optimal therapeutic approach.
Several studies recommended that surgery combined with radiotherapy may be the main treatment policy for limited and resectable NPACs, such as adenoid cystic carcinoma, mucoepidermoid carcinoma, and well-differentiated adenocarcinoma. ${ }^{6,17}$ A retrospective study by Liu et al ${ }^{16}$ revealed that the overall survival in T1-T2 patients was significantly improved comparing combined surgical treatment group ( $\mathrm{n}=12$ ) with the nonsurgical (radiotherapy) treatment group ( $\mathrm{n}=12 ; P=0.041)$. Multivariate analysis also indicated that surgical treatment was an independent predictor of survival in such patients $(P=0.017)$, which is consistent with the results of Guo et $\mathrm{al}^{4}$ and Liu et al. ${ }^{18}$ However, some studies revealed that the addition of PORT could not bring survival benefit for early-stage (stages I and II) patients with clear margin and without adverse prognostic factors such as lymphovascular or perineural invasion, which is a set of conditions usually restricted to low-grade variants..$^{7,19,20}$ On the basis of the patient's strong will and the cases reported in the literature, she had accepted PORT. Therefore, large multicentric studies or studies with large sample size should be conducted to further evaluate whether PORT is necessary or not for early stage lesions.

For the poorly differentiated or unresectable primary NPACs, regardless of the conventional and salivary gland type, it is very difficult to achieve the purpose of radical cure with surgery alone. Indications for definitive radiotherapy included early-stage tumors with positive surgical margin, poorly differentiated carcinoma, perineural invasion, and deep muscle invasion. Also, high-grade (poorly differentiated) adenocarcinomas are relatively radiosensitive, and radiotherapy or chemoradiotherapy is currently considered as the primary treatment policy for such patients. ${ }^{17}$ Chemotherapy was also administered to patients, but no obvious benefit of treatment was found in some studies. ${ }^{16,21}$ No matter how rare this tumor is, further studies are still needed to ensure the efficacy of chemotherapy.

Our case presented as a T1N0M0 lesion was low-grade papillary NPAC, and the lesion was resected completely. Surgical resection is the mainstay of treatment for limited or resectable lesions, and complete resection can achieve excellent prognosis. In some studies, follow-up data have not reported local recurrence or metastatic disease ranging from 5 years to 20 years after complete surgical removal. ${ }^{3,7,9,10}$ In a study by Wenig et al, ${ }^{7}$ one patient failed primary radiotherapy treatment and subsequently underwent surgical resection as a salvage and had been free of disease over an 11-year period. ${ }^{7}$ The disease-free survival has no difference compared with those receiving surgery alone. It indicated the predominance 
of surgery in the treatment of LGNPPA. Therefore, although our case had only a 4-month of follow-up, we can still predict its excellent prognosis.

However, owing to anatomical limitations and poor exposure of the tumor in the nasopharynx, it is sometimes difficult to completely remove the tumor with an adequate safety margin, especially in cases of large infiltrative tumors. Therefore, adjuvant treatment was needed to manage such tumors, which cannot be removed completely. Unfortunately, low-grade papillary NPACs, which belong to the type of well-differentiated adenocarcinomas, have low sensitivity to conventional radiotherapy or chemoradiotherapy, and it became a problem to deal with incompletely removed tumors.

Several studies revealed that photodynamic therapy (PDT) is a new treatment modality for head and neck cancers, with satisfactory treatment responses and minimal complications. ${ }^{22-25}$ In a study by Wang et al, ${ }^{26}$ PDT combined with topical 5-aminolevulinic acid as an effective modality of the postoperative adjuvant therapy was successfully used in the patient to eradicate the residual disease, without compromising the quality of life of the patient. Primary papillary NPAC is able to become qualified candidate for PDT, due to the limited lesions and low incidence of lymph node metastasis. ${ }^{10,12}$ PDT may be a more suitable alternative method to be the adjuvant therapy for incompletely resectable tumors.

Controversy also exists regarding the prognosis about the two categories of primary NPACs. Pineda-Daboin et $\mathrm{al}^{9}$ reported that the common-type NPAC is usually maintained as a low-grade malignancy and is associated with a good prognosis, while the prognosis of salivary gland-type NPACs is weak overall. However, a study by Guo et $\mathrm{al}^{4}$ showed that patients with common-type or salivary gland-type NPAC had no significant differences in survival rates (63\% versus $69 \%$, respectively). A study by Liu et $\mathrm{al}^{27}$ showed that all patients with the conventional NPAC or salivary gland-type nasopharyngeal carcinoma, regardless of the early stage or advanced stage, had no significant differences in 5-year overall survival and disease-free survival. A possible reason for the difference in the results may be the small sample size enrolled in each of the three studies. Prospective studies with broad crosssection are needed to draw a definite conclusion.

\section{Conclusion}

Although primary NPACs are very rare, they should be included in the differential diagnosis of nasopharyngeal tumor, even in children. Inappropriate treatment will result in recurrence of the disease. Therefore, definitive diagnosis of primary NPACs, especially low-grade papillary NPACs, is of extraordinary significance. Moreover, it requires appropriate surgical management to ensure an excellent prognosis for the patient. For limited or resectable adenocarcinoma, combined surgery with radiotherapy may be the proper treatment policy. Radiotherapy or chemoradiotherapy is suitable for the poorly differentiated NPACs or unresectable tumors. PDT may be a more suitable alternative method to be the adjuvant therapy for incompletely resectable tumors. Therefore, large multicentric studies or similar meta-analyses are necessary to evaluate further the optimal treatment approaches and the most relevant prognostic factors for these lesions.

\section{Disclosure}

The authors report no conflicts of interest in this work.

\section{References}

1. Wei WI, Sham JS. Nasopharyngeal carcinoma. Lancet. 2005; 365(9476):2041-2054.

2. Kuo T, Tsang NM. Salivary gland type nasopharyngeal carcinoma: a histologic, immunohistochemical, and Epstein-Barr virus study of 15 cases including a psammomatous mucoepidermoid carcinoma. Am J Surg Pathol. 2001;25(1):80-86.

3. He JH, Zong YS, Luo RZ, Liang XM, Wu QL, Liang YJ. Clinicopathological characteristics of primary nasopharyngeal adenocarcinoma. Ai Zheng. 2003;22(7):753-757.

4. Guo ZM, Liu WW, He JH. A retrospective cohort study of nasopharyngeal adenocarcinoma: a rare histological type of nasopharyngeal cancer. Clin Otolaryngol. 2009;34(4):322-327.

5. Tang Q, Hu QY, Piao YF, Hua YH, Fu AF. Clinical analysis of twentythree nasopharyngeal adenocarcinoma patients. Chin J Cancer Prev Treat. 2009;14(21):1669-1672.

6. Schramm VL Jr, Imola MJ. Management of nasopharyngeal salivary gland malignancy. Laryngoscope. 2001;111(9):1533-1544.

7. Wenig BM, Hyams VJ, Heffner DK. Nasopharyngeal papillary adenocarcinoma. A clinicopathologic study of a low-grade carcinoma. Am J Surg Pathol. 1988;12(12):946-953.

8. Ohe C, Sakaida N, Tadokoro C, et al. Thyroid-like low-grade nasopharyngeal papillary adenocarcinoma: report of two cases. Pathol Int. 2010; 60(2):107-111.

9. Pineda-Daboin K, Neto A, Ochoa-Perez V, Luna MA. Nasopharyngeal adenocarcinomas: a clinicopathologic study of 44 cases including immunohistochemical features of 18 papillary phenotypes. Ann Diagn Pathol. 2006;10(4):215-221.

10. Carrizo F, Luna MA. Thyroid transcription factor-1 expression in thyroid-like nasopharyngeal papillary adenocarcinoma: report of 2 cases. Ann Diagn Pathol. 2005;9(4):189-192.

11. Fu CH, Chang KP, Ueng SH, Wu CC, Hao SP. Primary thyroid-like papillary adenocarcinoma of the nasopharynx. Auris Nasus Larynx. 2008; 35(4):579-582.

12. van Hasselt CA, $\mathrm{Ng} \mathrm{HK}$. Papillary adenocarcinoma of the nasopharynx. J Laryngol Otol. 1991;105(10):853-854.

13. Xu T, Li ZM, Gu MF, et al. Primary nasopharyngeal adenocarcinoma: a review. Asia Pac J Clin Oncol. 2012;8(2):123-131.

14. Lee AW, Ma BB, Ng WT, Chan AT. Management of nasopharyngeal carcinoma: current practice and future perspective. J Clin Oncol. 2015; 33(29):3356-3364.

15. Petersson F, Vijayadwaja D, Loh KS, Tan KB. Reticular and myxoid non-keratinizing nasopharyngeal carcinoma: an unusual case mimicking a salivary gland carcinoma. Head Neck Pathol. 2014;8(3):364-368. 
16. Liu TR, Chen FJ, Qian CN, et al. Primary salivary gland type carcinoma of the nasopharynx: therapeutic outcomes and prognostic factors. Head Neck. 2010;32(4):435-444.

17. Liu TR, Chen FJ, Zhang GP, Yang AK. Different therapeutic strategies in primary salivary gland-type nasopharyngeal carcinomas. Curr Opin Otolaryngol Head Neck Surg. 2011;19(2):87-91.

18. Liu TR, Yang AK, Guo X, et al. Adenoid cystic carcinoma of the nasopharynx: 27-year experience. Laryngoscope. 2008;118(11): 1981-1988.

19. Terhaard CH, Lubsen $\mathrm{H}$, Rasch CR, et al. The role of radiotherapy in the treatment of malignant salivary gland tumors. Int J Radiat Oncol Biol Phys. 2005;61(1):103-111.

20. Terhaard CH, Lubsen H, Van der Tweel I, et al; Dutch Head and Neck Oncology Cooperative Group. Salivary gland carcinoma: independent prognostic factors for locoregional control, distant metastases, and overall survival: results of the Dutch head and neck oncology cooperative group. Head Neck. 2004;26(8):681-692.

21. Li HW, Cao JZ, Zhang QX. Clinical study of primary nasopharyngeal adenocarcinoma. Chin J Radiat Oncol. 2006;15(3):195-196.
22. Lou PJ, Jager HR, Jones L, Theodossy T, Bown SG, Hopper C. Interstitial photodynamic therapy as salvage treatment for recurrent head and neck cancer. Br J Cancer. 2004;91(3):441-446.

23. Lou PJ, Jones L, Hopper C. Clinical outcomes of photodynamic therapy for head-and-neck cancer. Technol Cancer Res Treat. 2003;2(4): 311-317.

24. Biel MA. Photodynamic therapy and the treatment of head and neck neoplasia. Laryngoscope. 1998;108(9):1259-1268.

25. D'Cruz AK, Robinson MH, Biel MA. mTHPC-mediated photodynamic therapy in patients with advanced, incurable head and neck cancer: a multicenter study of 128 patients. Head Neck. 2004;26(3):232-240.

26. Wang CP, Chang YL, Chen CT, Yang TH, Lou PJ. Photodynamic therapy with topical 5-aminolevulinic acid as a post-operative adjuvant therapy for an incompletely resected primary nasopharyngeal papillary adenocarcinoma: a case report. Lasers Surg Med. 2006;38(5):435-438.

27. Liu LZ, Zhang YM, Chen Y, Li L. Spreading patterns, prognostic factors and treatment outcomes of nasopharyngeal papillary adenocarcinoma and salivary gland-type carcinomas. Clin Otolaryngol. 2016;41(2): $160-168$.

\section{Publish your work in this journal}

OncoTargets and Therapy is an international, peer-reviewed, open access journal focusing on the pathological basis of all cancers, potential targets for therapy and treatment protocols employed to improve the management of cancer patients. The journal also focuses on the impact of management programs and new therapeutic agents and protocols on

\section{Dovepress}

patient perspectives such as quality of life, adherence and satisfaction. The manuscript management system is completely online and includes a very quick and fair peer-review system, which is all easy to use. Visit http://www.dovepress.com/testimonials.php to read real quotes from published authors. 